\title{
Evaluation of specific value of endoscopic biopsies and brush cytology for malignancies of the oesophagus and stomach
}

\author{
L. Witzel, F. HALTER ${ }^{1}$, P. A. GRETillat, U. SCHEURER, AND M. KELLER \\ From the Gastrointestinal Unit and Institute of Pathology, University of Berne, Berne, Switzerland
}

SUMMARY The value of multiple biopsies and brush cytology at oesophago-gastroscopy was assessed in relation to macroscopy and localization on 100 verified tumours in a prospective study. The cumulative accuracy achieved was $96 \%$. This was significantly better $(P<0.01)$ than that of biopsy $(83 \%)$ and of cytology $(85 \%)$. While the reliability of both procedures was not significantly different in malignancies of the oesophagus, the gastric body, and the antrum, cytology was significantly more accurate in cancers of the cardia $(90 \%$ and $55 \%$ respectively, $\mathrm{P}<0.05)$. Cytology was also more reliable in stenosing tumours $(92 \% / 72 \%, \mathrm{P}<0.05)$. In polypoid malignancies a positive but not significant trend was found in favour of multiple biopsies $(94 \% / 64 \%)$. One of the two early cancers was only diagnosed by cytology. The results confirm the high diagnostic accuracy of multiple endoscopic biopsies combined with brush cytology and demonstrate the value of cytology in stenosing tumours, especially in those of the cardia.

The reported rate of accuracy of multiple endoscopic biopsies and of brush cytology is at great variance. False negative results were observed between $7 \%$ and $68 \%$ for biopsy (Dollinger, 1972; Hampel et al., 1974) and $4 \cdot 2 \%-56 \%$ for brush cytology (Kobayashi et al., 1970; Dollinger, 1972). In the light of the difficulties in deciding whether an endoscopically visualized lesion is benign or malignant and the high value of a correct preoperative diagnosis, it was considered to be important to assess the reliability of both procedures in a prospective study. Also little is known of the value of both procedures in relation to the macroscopic aspect and the localization of an individual lesion and this question was therefore included in the protocol.

\section{Methods}

Two hundred and fifty-one patients with endoscopically suspect oesophageal (58) and gastric (193) lesions were investigated. The age of the 167 male and 84 female patients varied between 22 and 88 years (mean 57.8 years). Thirty-one had two ex-

'Address for correspondence: Dr F. Halter, Gastroenterologische Abteilung, Inselspital, 3010 Berne, Switzerland.

Received for publication 20 January 1976 aminations, 27 had three, six had four, and three had five. The observations in the tables relate to the initial endoscopy only.

After an overnight fast and premedication with 5-10 mg diazepam intramuscularly, a fibreoptic gastroduodenoscope type ACMI FO-7089 $\mathrm{P}$ or Olympus $\mathrm{GIFO}_{2}$ was introduced. The lesion was first biopsied and six to 10 tissue samples were taken using the standard biopsy forceps. The material was immediately fixed in $4 \%$ formaldehyde.

A standard type Olympus or ACMI cytology brush was then introduced through the biopsy channel of the instrument and the total area of the lesion was vigorously brushed. The brush was removed and the tip rinsed in approximately $10 \mathrm{ml}$ saline. This suspension was centrifuged within two to three hours after sampling at $3000 \mathrm{rpm}$ for 10 minutes. The sediments were distributed on slides, fixed with Delaunay solution and stained according to the Papanicolaou technique. Biopsy material and cytology specimens were examined in two independent laboratories by different investigators.

According to the macroscopic assessment and the subjective impression of four experienced investigators, the lesions were graded in five categories: (1) suspicion very low, (2) suspect, and (3) very suspect lesions, (4) definitely malignant lesions, and (5) no differentiation possible. 


\begin{tabular}{|c|c|c|c|c|c|c|c|}
\hline & \multirow[t]{2}{*}{ Total } & \multirow[t]{2}{*}{ Operation } & \multirow[t]{2}{*}{ Necropsy } & \multicolumn{2}{|l|}{ Follow-up } & \multirow{2}{*}{$\begin{array}{l}\text { Biopsy from } \\
\text { metastases }\end{array}$} & \multirow{2}{*}{$\begin{array}{l}\text { No } \\
\text { follow-up }\end{array}$} \\
\hline & & & & Endoscopic & Clinical & & \\
\hline \multicolumn{8}{|l|}{ Carcinoma } \\
\hline Oesophageal & 27 & 6 & 1 & & 18 & 2 & $\mathbf{0}$ \\
\hline Gastric & 70 & 58 & 3 & & 6 & 3 & 0 \\
\hline Gastric sarcoma & 3 & 2 & 1 & & & & \\
\hline Gastric ulcer & 95 & 32 & 2 & 49 & & & 12 \\
\hline Reflux oesophagitis & 31 & 7 & $\hat{1}$ & 19 & & & 4 \\
\hline Atypical gastric fold & 11 & & & 7 & 1 & & 3 \\
\hline Erosion & 6 & & & 3 & & & 3 \\
\hline Scar & 4 & & & 2 & & & 2 \\
\hline Operated stomach & 4 & & & 4 & & & \\
\hline Total & 251 & 105 & 8 & 84 & 25 & 5 & 24 \\
\hline
\end{tabular}

Table 1 Final diagnosis in 251 patients suffering from oesophageal (58) and gastric (193) lesions

\section{Results}

The final diagnosis of the lesions found at endoscopy are summarized in Table 1. Furthermore, information is given on how the diagnosis was verified. Twenty-seven of 100 tumours -97 carcinomas, three sarcomas-were localized in the oesophagus, 20 at the cardia, 26 in the gastric body, 21 in the antrum, and six within an operated stomach (Table 2). Eighty-three were verified by multiple biopsies, 85 by brush cytology. The cumulative accuracy reached $96 \%$, and was significantly better than that of either procedure alone $\left(\mathrm{P}<0.01, \chi^{2}\right.$ test). Both histology and cytology were negative in one oesophageal tumour and three carcinomas of the gastric body. In tumours of the cardia, the accuracy of the biopsy results was significantly smaller than that of cytology $\left(55 \% / 90 \%, P<0.05, \chi^{2}\right.$ test $)$. In all the other localizations the differences between the yield of either technique were not significantly different.

\begin{tabular}{lcccll}
\hline & Total & $M B$ & $B C$ & $M B+B C$ & Failure \\
\hline Oesophagus & 27 & 25 & 24 & 26 & 1 \\
Cardia & 20 & 11 & 18 & 20 & \\
Body and fundus & 26 & 21 & 20 & 23 & 3 \\
Antrum & 21 & 20 & 17 & 21 & \\
Recurrences in & & 6 & 6 & 6 & \\
$\quad$ operated stomach & 6 & 6 & 85 & 96 & 4 \\
Total & 100 & 83 & 85 & \\
\hline
\end{tabular}

Table 2 Diagnostic accuracy of multiple biopsies $(M B)$ and brush cytology $(B C)$ in 100 verified malignancies according to localization

An analysis of the data in respect of macroscopy (Table 3) gives significantly better results from cytology in 39 stenosing tumours with a diagnostic accuracy of $92 \%$ as compared with $72 \%$ for biopsy (P $<0.05, \chi^{2}$ test). In the 17 polypoid tumours, there was a positive trend in favour of the biopsy (diag-

\begin{tabular}{lcccl}
\hline & Total & $M B$ & $B C$ & Failure \\
\hline Stenosing & 39 & 28 & 36 & 1 \\
Tumourous ulcerated & 26 & 26 & 23 & \\
Polypoid tumourous & 17 & 16 & 11 & \\
Ulcerated & 12 & 9 & 10 & 2 \\
Elevated & 3 & 2 & 2 & 1 \\
Scar, erosion & 3 & 2 & 3 & \\
Total & 100 & 83 & 85 & 4 \\
\hline
\end{tabular}

Table 3 Diagnostic accuracy of multiple biopsies (MB) and brush cytology $(B C)$ in 100 verified malignancies tabulated according to growth

\begin{tabular}{lrrrrr}
\hline Endoscopic appearance & \multicolumn{4}{l}{ Final diagnosis } \\
\cline { 2 - 6 } & \multicolumn{3}{c}{ Benign } & \multicolumn{3}{c}{ Malignant } \\
& \multicolumn{1}{c}{ (no.) } & $(\%)$ & $($ no. $)$ & $(\%)$ \\
\hline No differentiation & 8 & $6 \cdot 3$ & 4 & 4 \\
Suspicion very low & 79 & $62 \cdot 2$ & 6 & 6 \\
Lesion & 27 & $21 \cdot 3$ & 6 & 6 \\
$\quad$ Suspect & 13 & $10 \cdot 2$ & 64 & 64 \\
$\quad$ Very suspect & 0 & 0 & 20 & 20 \\
$\quad$ Malignant & 127 & 100 & 100 & 100 \\
\hline
\end{tabular}

Table 4 Endoscopic appearance correlated with final diagnosis

nostic yield $94 \%$ as compared with $64 \%$ ), but the difference was not significant $\left(\mathbf{P}<0 \cdot 1, \chi^{2}\right.$ test).

In Table 4, the endoscopic aspect is correlated with the final diagnosis; $10.2 \%$ of those with a high suspicion were benign lesions. On the other hand, $12 \%$ with very low or low suspicion were malignant. In one out of the two early cancers only cytology was positive. In two ulcerative tumours with a positive cytology malignancy was not verified; however, in one of the two a complete histological examination of the operative specimen was not possible due to a technical error.

\section{Discussion}

The results of this study underline that a reliable classification of endoscopically visualized findings is 
not possible on the macroscopic aspect in a considerable proportion of eosophago-gastric lesions. An adequate diagnosis is, however, of vital importance in small tumours, especially in the noninvasive, so-called early cancers. Two such lesions observed in this study were not diagnosed as malignancies and were classified macroscopically as benign ulceration and erosion respectively.

The $83 \%$ diagnostic yield of multiple biopsies matches well with that of other series and demonstrates the high accuracy that can be achieved by endoscopic biopsy in modern fibreoptic endoscopy. In a review of 11 authors, seven had a diagnostic accuracy between $84 \%$ and $92 \%$ for biopsy and the average value for this method was $87 \%$ (Hermanek, 1973). The data show, however, that additional and significantly better information can be gained by combining biopsy sampling with brush cytology. One of the two early carcinomas was identified only by brush cytology. In stenosing tumours, especially in those of the cardia, the diagnostic accuracy of brush cytology is significantly higher than that of multiple biopsies. This simple technique neither prolongs examinations significantly nor does it increase the risk of the procedure. Until now, there have been no reports of complications of brush cytology (Witte, 1972). The diagnostic yield of cytology certainly depends on the skill of the examiner but, in addition, the sampling technique and the processing of the material are of the utmost importance. Lavage cytology is a time-consuming and less accurate procedure and the extra information gained with this technique in our hands was not significant (Witzel et al., 1974). The incidence of false positive results varies in the literature from 0.25-6.5\% (Prolla et al., 1972; Rachail et al., 1974; Weidenhiller, 1974). It is small in this study and it should be remembered that a negative finding in an operative specimen does not exclude an early carcinoma unless the material is subjected to a very detailed analysis.

\section{References}

Dollinger, H. (1972). Zur endoskopischen Beurteilung neoplastischer Wandveränderungen an Ösophagus und Magen. Leber Magen Darm, 2, 139-141.

Hampel, K. E., Stopik, D., and Niedobitek, F. (1974). Der diagnostische Wert von gezielten Biopsien bei Magentumoren. Zeitschift für Gastroenterologie, 12, 315-320.

Hermanek, P. (1973). Gastrobiopsy in cancer of the stomach. Endoscopy, 5, 144-147.

Kobayashi, S., Prolla, J. C., and Kirsner, J. B. (1970). Brushing cytology of the esophagus and stomach under direct vision by fiberscopes. Acta Cytologica, 14, 219-223.

Prolla, J. C., Xavier, R. G., and Kirsner, J. B. (1972). Exfoliative cytology in gastric ulcer. Its role differentiation of benign and malignant ulcers. Gastroenterology, 16, 3337.

Rachail, M., Rachail-Arnoux, M., Colomb, M., and Mouriquand, C. (1974). Le cytodiagnostic gastrique et oesophagien sous controle endoscopique. Lyon Médical, 231, 325-332.

Weidenhiller, S. (1974). Gastroenterologische Zytodiagnostik. Deutsche Medizinische Wochenschrift, 99, 8-11.

Witte, S. (1972). Die zytologische Tumordiagnostik. Fortschritte der Medizin, 90, 366-368.

Witzel, L., Halter. F. Grétillat, P. A., Scheurer, U., Wanger, F., and Blank, H. R. (1974). Diagnostische Wertigkeit von Biopsie, Bürsten- und Spülzytologie bei endoskopischem Verdacht auf maligne Oesophagusoder Magenaffektionen. Presented at the annual meeting of the Schweizerische Gesellschaft für Gastroenterologie, Freiburg, September 19-21. 